
allemande

\title{
Vom Umgang mit Katastrophen
}

\section{Klaus Vondung}

\section{CpenEdition}

\section{Journals}

Édition électronique

URL : https://journals.openedition.org/allemagne/2177

DOI : 10.4000/allemagne. 2177

ISSN : 2605-7913

\section{Éditeur}

Société d'études allemandes

\section{Édition imprimée}

Date de publication : 1 juillet 2020

Pagination : $9-15$

ISSN : 0035-0974

\section{Référence électronique}

Klaus Vondung, Vom Umgang mit Katastrophen" Revue d'Allemagne et des pays de langue allemande [Online], 52-1 | 2020, Online erschienen am: 06 Juli 2021, abgerufen am 20 Februar 2022. URL: http:// journals.openedition.org/allemagne/2177 ; DOI: https://doi.org/10.4000/allemagne.2177 


\section{Préface}

\section{Vom Umgang mit Katastrophen}

\section{- Klaus Vondung*}

Machen sich die Deutschen mehr Sorgen um Klima und Umwelt als andere Nationen? Das ist nicht sicher. Aber es gibt Indizien. Dass im Englischen das einschlägige Stichwort unübersetzt bleibt, also von "German Angst“ die Rede ist, soll wohl zum Ausdruck bringen, dass Engländer und Amerikaner diese „Angst“ für typisch deutsch und außerdem für etwas übertrieben halten. Auch unsere Nachbarn im Westen machen sich offenbar weniger Sorgen. Dies zeigte sich schon 1986, nach dem Reaktorunfall von Tschernobyl. In Deutschland ließ man eine Zeitlang die Kinder nicht mehr draußen im Sandkasten spielen und aß keinen Kopfsalat. Pilzsucher (zu denen ich hobbymäßig gehöre) diskutierten leidenschaftlich, welche Pilze „dumm“ seien, weil sie unterschiedslos Schwermetalle, darunter radioaktives Caesium, dem Boden entnehmen (z. B. Maronen), im Gegensatz zu "gescheiten Pilzen“, die das nicht tun (wie z. B. Steinpilze). Aus französischer Sicht endete wohl die radioaktive Wolke, die sich von Tschernobyl nach Westen ausdehnte, am Rhein. Ein Leitartikel in der französischen Zeitschrift Documents, die sich vorwiegend mit „deutschen Fragen“ beschäftigt, brachte die unterschiedlichen Reaktionen auf den Gegensatz „deutscher Emotionen und französischer Gleichgültigkeiten“(1).

Noch eklatanter waren die Reaktionen auf die Katastrophe von Fukushima, auf das Erdbeben vom 11. März 2011, den Tsunami und den dadurch bewirkten GAU im dortigen Atomkraftwerk. Die deutsche Bundeskanzlerin Angela Merkel sprach schon am 14. März vom „apokalyptischen Ausmaß der Zerstörung“ und erklärte: „Das verändert die Lage auch in Deutschland“(2). Einige Tage später beschloss die Regierung, die sieben ältesten Atommeiler abzuschalten. Die Absicht, vollständig aus der Atomenergie auszusteigen, fand Unterstützung in allen Parteien. Am 30. Juni wurde im

\footnotetext{
* Professor Emeritus für Germanistik / Neuere Literaturwissenschaft an der Universität Siegen.

1 „L'apocalypse atomique. Émotions allemandes, indifférences françaises?", Documents. Revue des questions allemandes, 41/2 (1986), S. $3 \mathrm{ff}$.

2 Der Stern, Nr. 12, 17.03.2011, S. 70.
} 
Bundestag das Atomgesetz novelliert. Die große Mehrheit der Abgeordneten beschloss den vollständigen Atomausstieg. Wie dramatisch die Abkehr von der Atomenergie ablief, kann man daran ermessen, dass nur viereinhalb Monate vor der Katastrophe von Fukushima der Bundestag mit der Mehrheit der Regierungskoalition von CDU und FDP noch eine Laufzeitverlängerung der Atomkraftwerke um durchschnittlich zwölf Jahre beschlossen hatte.

Die Nachbarn Deutschlands tun sich schwerer mit dem Ausstieg aus der Atomenergie. Symptomatisch ist der Umgang mit störanfälligen Reaktoren. Das älteste französische Atomkraftwerk Fessenheim im Elsass gilt als eine der unsichersten Nuklearanlagen in Europa; es kann eine Serie von Pannen aufweisen. Nach mehreren Demarchen der deutschen Regierung kündigte Präsident Macron Ende 2018 nun die Schließung des AKW für den Sommer 2020 an. Die belgische Atomanlage Tihange wurde nicht, wie geplant, 2015 vom Netz genommen, deren Laufzeit vielmehr trotz mehrerer Pannen um zehn Jahre verlängert. Tihange ist $70 \mathrm{~km}$ von Aachen entfernt. Der Versuch des Ministerpräsidenten von Nordrhein-Westfalen, eine frühere Abschaltung zu erreichen, war erfolglos. In den an die belgische Grenze anschließenden Landkreisen gibt es angeblich Pläne oder sogar schon Vorkehrungen, im Fall eines GAU oder sogar prophylaktisch die Bevölkerung mit Kaliumjodidtabletten zu versorgen. Laut einer Meldung der Süddeutschen Zeitung vom Februar 2019 forderten die deutschen Bundesländer von der Regierung „mehr Engagement gegen störanfällige Atomkraftwerke nahe der deutschen Grenze“. Exporte von Brennelementen an diese Anlagen sollten gestoppt werden ${ }^{(3)}$. Mittlerweile hat das Bundesumweltministerium einen Gesetzentwurf vorgelegt, dem zufolge „künftig in einem Radius von $150 \mathrm{~km}$ rund um Deutschland kein AKW mehr mit Brennstoffen beliefert werden dürfe, sofern es älter als 30 Jahre ist“(4).

Die Störanfälligkeit von Atomkraftwerken ist freilich nur ein Faktor bei den Überlegungen und gegebenenfalls Entscheidungen über sichere, preisgünstige und außerdem saubere Energieversorgung. Frankreich bezieht 75 Prozent seines Stroms aus Atomkraftwerken. Präsident Macron schob das Ziel, diesen Anteil auf 50 Prozent zu reduzieren, um zehn Jahre auf 2035 hinaus. Der Vorteil der Atomenergie ist ihre Sauberkeit, solange die Atomkraftwerke störungsfrei funktionieren und sieht man einmal vom Problem des zu entsorgenden Atommülls ab. Der hohe Anteil des Atomstroms in Frankreich im Vergleich zu Deutschland hat zur Folge, dass die CO2-Emissionen in Frankreich niedriger sind, obwohl sie auch dort ansteigen. In Deutschland produzieren über hundert Kraftwerke ausschließlich oder überwiegend Strom aus Braun- und Steinkohle; sie sind damit - laut WWF - verantwortlich für mehr als ein Drittel der CO2-Emissionen. Übertroffen wird dieser Ausstoß freilich noch durch die Emissionen, die durch die Verbrennung von Mineralöl entstehen, wobei der Verkehr die schlechteste Klimabilanz aufweist. Schon Mitte 2018 war klar, dass Deutschland das Ziel des Pariser Klima-Abkommens von 2015, die Erderwärmung im Vergleich zum vorindustriellen Zeitalter auf deutlich unter zwei Grad zu begrenzen, verfehlen wird ${ }^{(5)}$.

3 Süddeutsche Zeitung, 16./17.02.2019, S. 6; zur Schließung von Fessenheim Süddeutsche Zeitung, 28.11.2018, S. 7.

4 Süddeutsche Zeitung, 06.12.2019, S. 7.

5 www.wwf.de (08.05.2019); de.statista.com (08.05.2019). 
Die Katastrophe von Fukushima hatte in Deutschland unmittelbare politische Auswirkungen. Interessant ist aber auch, wie mit diesem Ereignis sprachlich umgegangen wurde. Berichte und Kommentare in Zeitungen und Zeitschriften benützten ständig den Begriff „Apokalypse“, um Erdbeben und Tsunami und den anschließenden GAU im AKW Fukushima zu bezeichnen. Und zahlreiche Politiker bedienten sich ebenfalls dieses Etiketts, von der Bundeskanzlerin über den damaligen bayerischen Ministerpräsidenten Seehofer bis zum früheren EU-Energiekommissar Günther Oettinger ${ }^{(6)}$.

Warum wurde der Begriff „Apokalypse“ so häufig gebraucht? Offenbar deswegen, weil er Untergang, Tod und Vernichtung in großem Maßstab bezeichnet. Vermutlich schwingt sogar die Assoziation an einen totalen und endgültigen Untergang der Welt mit. Jedenfalls drückten sich nicht nur die Interpretationen einer tatsächlichen Katastrophe in diesem Begriff aus, sondern immer wieder auch die Befürchtungen eines totalen Untergangs. Die Berichte des Weltklimarats der UN (IPCC) der vergangenen Jahre, die vor den Folgen der Klimaerwärmung warnten, wurden eine Zeitlang in der Presse gerne mit dem ominösen Begriff versehen, z. B.: „Droht die Apokalypse?“ oder: „Uno schlägt Alarm - Klima-Apokalypse naht“(7).

Die Assoziation zum Ende der Welt und der Menschheit, die der Begriff „Apokalypse“ evoziert, ergibt sich nicht zufällig. Denn die Schrift, die der abendländischen Tradition apokalyptischen Denkens den stärksten Anstoß gab und zugleich den Begriff bereitstellte, die Offenbarung (= Apokalypse) des Johannes, prophezeite tatsächlich den Untergang der Welt. Allerdings hatte es in dieser Schrift und in der ganzen religiösen Tradition damit nicht sein Bewenden. Der Apokalypse kommt es letztlich auf die neue Welt an, die dem Untergang der alten folgen soll, auf die Prophezeiung einer „neuen Erde“, eines „neuen Jerusalem“, wie es in der Offenbarung heißt. Die Apokalypse war eine Erlösungsvision. Dies blieb auch so in den weltlichen, politischen Spekulationen, die nach apokalyptischer Art ebenfalls eine neue Welt, nun ein „irdisches Paradies“ und einen „neuen Menschen“ schaffen wollten. Im Verlauf des 20. Jahrhunderts ging jedoch der Glaube verloren, man könne ein von Menschen geschaffenes Paradies errichten. Endgültig erledigt wurde dieser Glaube durch die Atombomben von Hiroshima und Nagasaki. Nur die erste Hälfte der apokalyptischen Vision, die Vernichtung der Welt, war zu machen; gerade weil dies nun möglich geworden war, verflüchtigte sich die zweite Hälfte der Vision, die Errichtung einer neuen, vollkommenen Welt. Was blieb, war allein der Untergang. Seither wird mit dem Begriff „Apokalypse“ nur noch Tod und Vernichtung assoziiert und auch die Angst vor dem Untergang der Welt durch Umweltzerstörung und Klimawandel zum Ausdruck gebracht.

Warum aber war und ist häufig immer noch „Apokalypse“ der Generaltitel für Katastrophen unterschiedlicher Art, für tatsächliche wie für befürchtete? Das Beispiel der „Apokalypse von Fukushima“ bietet Antworten. Da ist zunächst die Funktion der schlagwortartigen Zusammenfassung der Ereignisse. Mit der Verwendung des

6 Wörtliche Zitate und Belege bei Klaus Vondung, Apokalypse ohne Ende, Heidelberg, Winter, 2018, S. 21-23.

7 Spiegel Online, 02.02.2007; Frankfurter Allgemeine Zeitung, 19.11.2007; Focus, 07.07.2008, S. 82; Frankfurter Allgemeine Zeitung, 08.12.2011; vgl. Süddeutsche Zeitung, 14.04.2014. 
Begriffs „Apokalypse“ fand eine Einordnung statt und auf diese Weise fast so etwas wie Bewältigung. Wenn das Entsetzliche auf den Begriff gebracht wird, ist es zugleich dingfest gemacht und gebannt.

Eine weitere Funktion: Mit dem Begriff „Apokalypse“ sind Assoziationen verbunden - Zerstörung, Tod, Untergang -, die stark auf Emotionen wirken. Da in der Debatte nach dem 11. März 2011 der Begriff „Apokalypse“ Entsetzliches evozierte, verstärkte er bei vielen Menschen und eben auch bei den politischen Entscheidungsträgern in Deutschland den Willen, aus der Atomkraft auszusteigen; und er beförderte den Entschluss, auf den Ausstieg hinzuwirken. Die Beschwörung der „Apokalypse“, so könnte man sagen, hatte zumindest mittelbar den Untergang aufschiebende Wirkung ${ }^{(8)}$.

Leider gibt es keine vergleichbaren Effekte bei anderen Verursachern von möglichen Katastrophen. Mittlerweile gibt es wieder einen bedrohlichen Rüstungswettlauf und Kriegsgefahr im Nahen Osten; die Rüstungsexporte steigen kontinuierlich. Aber nur noch selten ist von einer drohenden „nuklearen Apokalypse“ die Rede ${ }^{(9)}$. Im Februar 2019 titelte die Süddeutsche Zeitung in einem Artikel über den Klimawandel: „Eine globale Umfrage zeigt, dass die Folgen der Erderwärmung mehr Menschen beunruhigen als die nukleare Bedrohung “(10). Und das Bedrohungspotential, das der Begriff „Apokalypse“ zum Ausdruck brachte, scheint verloren zu gehen und nicht mehr Antrieb zu sein, praktische Konsequenzen zu ziehen. Kurt Kister, Chefredakteur der Süddeutschen Zeitung, stellte in einem Abonnentenbrief fest, es würden „gerne und viel zu häufig Begriffe aus der Psychopathologie und der Apokalyptik benutzt [...], um Widrigkeiten einzuordnen. Ständige Übertreibung verringert leider die Glaubwürdigkeit“(11). Die Bild- und Medienwissenschaftlerin Birgit Schneider benützte zwar noch einmal den ominösen Begriff für den Titel eines ZEIT-Artikels über die Erderwärmung: „Die Apokalypse ist tiefrot“(12), wobei sie auf die Farbskalen anspielte, in denen die zunehmende Erderwärmung von blau zu rot dargestellt wird. Aber sie stellte für diese farblich eigentlich sehr eindrücklichen Warnungen denselben Effekt fest wie beim allzu häufigen Schlagwort-Gebrauch des Begriffs „Apokalypse“: „Das Handeln blockieren die roten Karten eher, weil sie den Klimawandel als unaufhaltsame Katastrophe oder gar als Weltende zeigen“(13).

Dennoch wird der Begriff „Apokalypse“ immer noch gern gebraucht, um ultimativen Schrecken zu verbreiten. Die Zeitschrift Der Spiegel bildete auf dem Titelblatt seiner Ausgabe vom 18. Mai 2019 den Kopf der Bundeskanzlerin vor schwarzem Hintergrund $\mathrm{ab}$ und untertitelte: „Nach ihr die Finsternis. Angela Merkels apokalyptischer Blick auf die Lage der Welt“. Im Leitartikel zum Titel wurde konstatiert, Merkels historische Vergleiche zur gegenwärtigen Weltlage „können apokalyptischer nicht sein“. Und es ist von der Hoffnung die Rede, „dass wenigstens die Kanzlerin

8 „Katechon“ im Sinne von Carl Schmitt; siehe K. Vondung, Apokalypse ohne Ende (Anm. 6), S. 30-32.

9 Süddeutsche Zeitung, „Gehört, gelesen, zitiert“, 12.02.2019, S. 11.

10 Süddeutsche Zeitung, „Klimawandel verbreitet die größte Angst“, 11.02.1019.

11 Kurt Kister, Brief aus der SZ-Chefredaktion, 15.02.2019.

12 Birgit Schneider, „Erderwärmung: Die Apokalypse ist tiefrot“, www.zeit.de/kultur/201905erderwärmung (07.05.2019).

13 Ebd., S. 2. 
versucht, die Reiter der Apokalypse aufzuhalten“(14), im Sinne der „Katechontik“ nach Carl Schmitt, des Hinausschiebens oder sogar Aufhaltens des Untergangs ${ }^{(15)}$. Aber entsprechendes Handeln wird vermisst.

Wie also überwindet man die „Apokalypseblindheit“, und: „Wie kommt man vom Wissen zum Handeln?“(16) Mit Blick auf den Klimawandel gibt es einige Zeichen der Hoffnung, die freilich noch keine Gewähr für wirkliche Besserung sind. Die Nutzung erneuerbarer Energien nimmt zu, wenn auch noch zu langsam. Kohlekraftwerke werden nach und nach geschlossen, wenn auch noch zu langsam. Als Alternative zu den Verbrennungsmotoren der Autos mit ihren CO2-Emissionen und dem Ausstoß von Feinstaub hat die Entwicklung von Hybrid- und Elektrofahrzeugen begonnen, aber bis zu einer spürbaren Größenordnung ist noch ein weiter Weg. Doch der Druck auf die Politik wächst. Die Schulstreiks, die von der 16-jährigen Schwedin Greta Thunberg initiiert wurden, veranlassten auch in Deutschland Tausende Schüler, unter dem Slogan „Fridays for Future“ die Schule zu schwänzen und gegen Erderwärmung und Artensterben und für die Schließung von Kohlekraftwerken zu demonstrieren. Die Schüler brachten damit nicht nur manche Lehrer und die Schulbehörden in Verlegenheit, sondern auch die Politik. Mittlerweile haben sich zahlreiche Wissenschaftler, Künstler und andere Prominente dieser Protestbewegung angeschlossen. Am 20. September 2019 fand ein weltweiter Klima-Streik statt mit anschließenden Aktionstagen.

Ein durchaus bemerkenswerter Erfolg war einer regionalen Aktion beschieden. Im Februar 2019 unterschrieben 1,8 Millionen Bürger Bayerns ein Volksbegehren „Rettet die Bienen“. Das bedeutete, dass fast jeder Fünfte wahlberechtigte Bayer das Volksbegehren unterschrieb. Eine Studie hatte ermittelt, dass in Deutschland während der letzten 27 Jahre die Insektenbiomasse um durchschnittlich 76 Prozent zurückgegangen ist $^{(17)}$. Der Erfolg des Volksbegehrens, dem der Entwurf eines Bündnisses für mehr Artenvielfalt zugrunde lag, hätte eine Volksabstimmung nach sich ziehen können. Um dies zu verhindern, nahm die bayerische Staatsregierung einen radikalen Kurswechsel in der Landwirtschaftspolitik vor und erklärte sich bereit, große Teile des Entwurfs für mehr Artenvielfalt zu übernehmen ${ }^{(18)}$. Mittlerweile, am 17. Juli 2019, beschloss der Bayerische Landtag ein entsprechendes Gesetz zum Schutz der Artenvielfalt. Und am 15. November 2019 verabschiedete der Deutsche Bundestag ein umfangreiches Klimaschutzgesetz.

Wie gesagt, es gibt einige Zeichen der Hoffnung. Aber nach wie vor überwiegen die schlechten Nachrichten. Der Weltklimarat hat festgestellt, dass der Ausstoß von Treibhausgasen weltweit weiter ansteigt. Dies ist auch in Deutschland der Fall. Viele Experten halten das Klimaschutzgesetz des Deutschen Bundestags für unzureichend. Erst jetzt wurde bekannt, dass das Umweltbundesamt, eine Behörde des Bundesumweltministeriums, schon im Juni 2019 ein Papier vorgelegt hat, in dem drastische

14 Der Spiegel, Nr. 21, 18.05.2019, S. 17f.

15 Siehe K. Vondung, Apokalypse ohne Ende (Anm. 6), S. 30-32; vgl. oben, Anm. 7.

16 B. SCHNEIDER, „Erderwärmung“ (Anm. 12), S. 4.

17 Süddeutsche Zeitung, „Biene und Co. sollen leben“, 18.02.2019, S. 5.

18 Süddeutsche Zeitung, 18.02.2019, S. 4; ebd., S. 5; Süddeutsche Zeitung, „Der erzwungene Kurswechsel“, 04.04.2019, S. 36 . 
Maßnahmen angeregt werden: eine hohe Abgabe auf CO2-Emissionen, und zwar eine wesentlich höhere als sie nun im Klimaschutzgesetz vorgesehen ist, eine Erhöhung der Maut für Lastkraftwagen, ein Tempolimit von 120 Stundenkilometern auf Autobahnen ${ }^{(19)}$. Die bisherige Leiterin des Umweltbundesamtes, Maria Krautzberger, hält das Klimaschutzgesetz für „viel zu zaghaft“, und meint: „Die Politik hat zu wenig Mut“(20).

Der Generalsekretär der Vereinten Nationen, António Guterres, eröffnete am 2. Dezember 2019 die Weltklimakonferenz in Madrid mit einem dramatischen Appell an die Weltgemeinschaft, den „Krieg gegen die Natur“ zu beenden. Konkrete Entscheidungen jedoch wurden nicht getroffen, trotz zweitägiger Verlängerung der Konferenz; zu verschieden sind die Interessen oder auch die Möglichkeiten der einzelnen Staaten. In der Abschlusserklärung vom 15. Dezember 2019 wurden die 196 teilnehmenden Staaten lediglich an die Zusage erinnert, bis zum Klimagipfel im nächsten Jahr die Klimaschutzziele für 2030 möglichst zu verschärfen. Der Generalsekretär der UN und Mitglieder des Weltklimarats reagierten enttäuscht ${ }^{(21)}$.

Einen Schritt weiter ist man (vielleicht) in der Europäischen Union. Die Kommissionspräsidentin, Ursula von der Leyen, stellte am 11. Dezember 2019 einen umfassenden Plan vor, die EU bis 2050 klimaneutral zu machen. Beim Treffen der Staats- und Regierungschefs der EU verständigte man sich zwei Tage später auf dieses Ziel. Polen allerdings, das 78 Prozent seiner Energie aus Kohle gewinnt, wollte sich auf dieses Ziel nicht verpflichten lassen. Ungarn und Tschechien behielten sich vor, Atomkraft als „saubere Energiequelle“ in ihrer jeweiligen Klimabilanz zu berücksichtigen. Welche Widerstände innerhalb der einzelnen Mitgliedsländer von Seiten der Industrie und der Landwirtschaft zu überwinden und welche Finanzierungsprobleme zu lösen sind, ist noch gar nicht abzusehen. Skepsis ist also angebracht.

Noch ist der Anstieg der Treibhausgase ungebremst. Das bedeutet, dass die Erwärmung des Weltklimas nicht gestoppt ist, dass arktisches Eis, Grönlandeis und Gletscher schmelzen, der Meeresspiegel steigt. Die Regenwälder in Brasilien und Indonesien werden weiterhin abgeholzt. Die Artenvielfalt geht zurück; 1 Million pflanzliche und tierische Arten sind vom Aussterben bedroht. Und dass die Umweltprobleme derzeit stärker im Fokus sind, darf nicht übersehen lassen, dass der Rüstungswettlauf wieder zugenommen hat und dass in verschiedenen Weltgegenden bewaffnete Konflikte wenn nicht gar Kriege drohen oder sogar im Gange sind.

Die Beschwörung einer drohenden „Apokalypse“ hilft nicht viel, wenn es beim Schlagwort bleibt, das nicht mehr schreckt oder sogar abstumpft. Funktional könnte das Schreckbild allenfalls sein, wenn es im Sinne des „Katechon“ dazu beitragen würde, sofortiges oder zumindest zügiges Handeln zu initiieren, das den Untergang aufhalten kann. Zu solchem Handeln wäre die Politik aufgerufen, aber auch - was das Aufhalten des Klimawandels anlangt - jeder einzelne von uns.

19 Süddeutsche Zeitung, 05.12.2019, S. 6.

20 Süddeutsche Zeitung, 12.12.2019, S. 5.

21 Süddeutsche Zeitung, 16.12.2019, S. 1 u. 2. 


\section{Zusammenfassung}

Der Begriff "Apokalypse" wird in Deutschland gern gebraucht, um große Katastrophen zu charakterisieren. Journalisten wie Politiker bezeichneten $z$. B. die Katastrophe von Fukushima 2011 als „apokalyptisch“. Aber auch befürchtete künftige Katastrophen werden oft mit diesem Schlagwort versehen. Zur Zeit sind dies die befürchteten Auswirkungen des Klimawandels, Vergiftung der Atmosphäre, Erwärmung des Weltklimas, Dürreperioden, Hungersnöte. Der Begriff „Apokalypse“ ruft Assoziationen zu Zerstörungen großen Ausmaßes, zu Tod und Untergang hervor, lässt sogar an das Weltende denken. Er wird auch deswegen gebraucht, um aufzurütteln und Maßnahmen anzustoßen, die den Untergang aufhalten können. Andererseits birgt der allzu häufige Gebrauch von Schreckensbildern die Gefahr der Abstumpfung. Es ist noch nicht ausgemacht, ob die Warnungen vor den Folgen des Klimawandels zu wirkungsvollem politischen Handeln und zu Verhaltensänderungen der einzelnen Menschen führen werden.

\section{Résumé}

Le concept d' "apocalypse" est fréquemment utilisé en Allemagne pour caractériser des catastrophes de grande ampleur. Journalistes et hommes politiques ont par exemple qualifié la catastrophe de Fukushima en 2011 d' "apocalyptique». Mais les peurs au sujet de catastrophes à venir sont également fréquemment associées à ce mot-clé. Actuellement, cela concerne les conséquences redoutées du changement climatique, l'empoisonnement de l'atmosphère, le réchauffement du climat mondial, les périodes de sécheresse, les famines. Le concept d' "apocalypse» est associé à des destructions de grande ampleur, à la mort et au déclin, il fait même penser à la fin du monde. On l'utilise également pour réveiller les consciences et inciter à prendre des mesures qui seraient à même d'arrêter le déclin. D’un autre côté, l'évocation trop fréquente de scénarioscatastrophes porte en elle le danger d'une désensibilisation. Rien ne nous garantit que les mises en garde contre les conséquences du changement climatique finiront par conduire les hommes à une action politique efficace et à un changement individuel de leur comportement.

\footnotetext{
Abstract

The term "apocalypse" is frequently used in Germany for characterizing big catastrophes. Journalists as well as politicians, for instance, described the catastrophe of Fukushima in 2011 as "apocalyptic". This catchword is being used also for catastrophes that are expected in the future. At present, one fears the future effects of the climate change, the pollution of the atmosphere, the warming-up of the climate, periods of drought, famine. The term "apocalypse" elicits associations of grand devastations, of death and destruction, even of the end of the world. The term is also used in order to stir up and induce measures that could prevent these effects. On the other hand, the frequent use of dreadful images and visions involves the danger that people become bunted and indifferent. It is still undecided whether or not the warnings of the climate change will lead to effective political actions and to a change of behavior.
} 